\title{
Optical characterization of thin Au films by standard and polaritonic ellipsometry
}

\author{
N.L. Dmitruk, O.V. Fursenko, O.S. Kondratenko, V.R. Romanyuk \\ Institute of Semiconductor Physics, NAS of Ukraine, 45 prospect Nauky, 03028 Kyiv, Ukraine \\ Phone: +38(044) 265 6486; fax: +38(044) 265 8342; e-mail: nicola@dep39.semicond.kiev.ua
}

\begin{abstract}
This work is aimed at optical characterization of thin Au films by multiple-angleof-incidence reflectance ellipsometry at the fixed wavelength $(632.8 \mathrm{~nm})$ in standard and attenuated total reflection (ATR) modes in contact with different dielectric media (water, alcohol and air). The comparative experimental evaluation of the precision of determined optical parameters (refractive index $n$ and absorption coefficient $k$ ) and thickness for both ellipsometric modes has been studied by calculation of their sensitivity correlation coefficients. Mediumand mode-related optical constants of Au films were revealed. In the ATR mode the effective optical parameters were higher then in the standard mode, $n$ decreased and $k$ increased with increasing the refractive index of adjoining medium. This effect must be taken into consideration in polaritonic optoelectronic and optochemical sensor technique.
\end{abstract}

Keywords: ellipsometry, ATR, polariton, optical constants, Au, surface plasmon.

Paper received 12.05.03; accepted for publication 17.06.03.

\section{Introduction}

Nowadays the interest to thin semitransparent metal films is strongly increased due to their applications for polaritonic optoelectronics and optochemical sensor technique. The optical constants (refractive index $n$ and absorption coefficient $k$ ) of these films need to be known. More often they are obtained for thick films or bulk materials from reflection measurements in standard (external) mode in air atmosphere or in-situ, to avoid surface oxidation [1]. However, the optical constants of extremely thin films differ from those of bulk materials [2, 3]. Often their accurate and unambiguous determination simultaneously with the thickness $(d)$ by optical methods is difficult. One of the reasons is incorrect minimizing of the least squares functional due to the existence of strong correlation between parameters and errors in the initial data. It is known that the precision of the attenuated total internal reflection (ATR) mode utilizing the excitation of surface polaritons is higher in comparison with the standard mode in thin film analysis, due to the resonant enhancement of electromagnetic fields [4-7]. Application of the ATR mode in ellipsometry gives a possibility to increase the sensitivity of measurements to the param- eters of metal films [6]. But, at the same time, there are some published results showing that the dielectric function describing the optical properties of metal films are influenced by immersion in different dielectric media $[8,9]$.

In this paper, the optical constants of thin Au films were obtained in two modes of multiple-angle-of-incidence (MAI) reflectance ellipsometry: standard mode with external reflection and polaritonic mode with attenuated total internal reflection in contact with different dielectric media. This enabled us to compare the reliability of determination of optical parameters and thickness of thin absorbing films. Differences of the optical parameters of $\mathrm{Au}$ films were revealed in the course of measurements using both ellipsometric modes. In the ATR mode, the adjacent medium-related optical constants were obtained. The reasons of this are discussed.

\section{Experimental details}

Au films up to $50 \mathrm{~nm}$ thick with an ultrathin Cr underlayer for Au adhesion were thermally evaporated in vacuum at room temperature on the bottom of a $68^{\circ}$ trapezoidal glass prisms $(n=1.5)$ to realize the Kretschman geometry for ATR measurements [5]. The deposition rate was 
$1.0-1.5 \mathrm{~nm} / \mathrm{sec}$. The "mass thickness" and "local thickness" of films were controlled by a quartz resonator during the deposition process and by AFM (Nanoscope IIIA, Digital Instruments, USA) just after deposition, accordingly. In the latter case, the step height between the covered by $\mathrm{Au}$ and uncovered surface of the glass substrate was measured (Fig. 1c). The morphology of the film and its geometrical parameters have been determined (Fig. 1, a, b).

MAI ellipsometry measurements were done using LEF-3M null-ellipsometer (Feodosia, Ukraine) with a fixed compensator and He-Ne laser as a light source $(\lambda=$ $=632.8 \mathrm{~nm})$. Two modes: standard and ATR in contact with different dielectric media (air $(n=1)$, water $(n=$ $=1.333), 20 \%$-alcohol $(n=1.345)$ and $100 \%$-alcohol $(n=$ $=1.361$ ) were used. The investigated incident angle range was in the vicinity of the Brewster angle in the case of standard ellipsometry, and near the surface plasmon resonance angle for ATR ellipsometry, taking into account the instrument's limited angle range. By fitting the experimental curves of ellipsometric angles $\Psi(\varphi)$ and $\Delta(\varphi)$ to theoretical ones using a structural model ambient/film/ substrate, the effective optical parameters and thickness of $\mathrm{Au}$ films have been calculated under assumption of their isotropy.

\section{Results and discussion}

As follows from AFM results (Fig. $1 \mathrm{a}, \mathrm{b}$ ), the $5 \mathrm{~nm}$ Au film consisted of separate islands; the $15 \mathrm{~nm}$ film was nearly continuous with a nanorelief of about $3 \mathrm{~nm}$, and the $45 \mathrm{~nm}$ film (not shown here) was entirely continuous with the same value of nanorelief of $3 \mathrm{~nm}$. Dependencies of the ellipsometric parameters $\Psi(\varphi)$ and $\Delta(\varphi)$ for these films, measured in standard and ATR modes with air and water as contacting media, are shown in Fig. 2 (a, b). In the case of ATR with water, the surface plasmon polariton resonance detected by both a dip in the $\Psi(\varphi)$ and the sharp "oscillation" in the $\Delta(\varphi)$ dependencies was observed. In the case of ATR with air, the optimal angle range corresponding to the surface plasmon excitation was outside the measurement angle range due to instrument's limitation.

The uniqueness problem of the obtained values of a thickness and optical constants is one of the main problems in ellipsometry. Non-unique results indicate that two or more of the parameters in the model are strongly correlated, and the parameter set must be reduced in order to eliminate the correlation. A comparative quantitative evaluation of the precision of the conductive film parameter determination by different ellipsometry modes has been performed by calculation of the sensitivity correlation coefficients [10] between the actual parameters. In the Table 1 the two-parameter correlation coefficients are shown in different measurement modes (Fig. 2). As expected, the correlation between parameters in the ATR mode was less then in the standard mode. The ATR regime with water has superiority over ATR with air providing a better accuracy of parameter determination. The
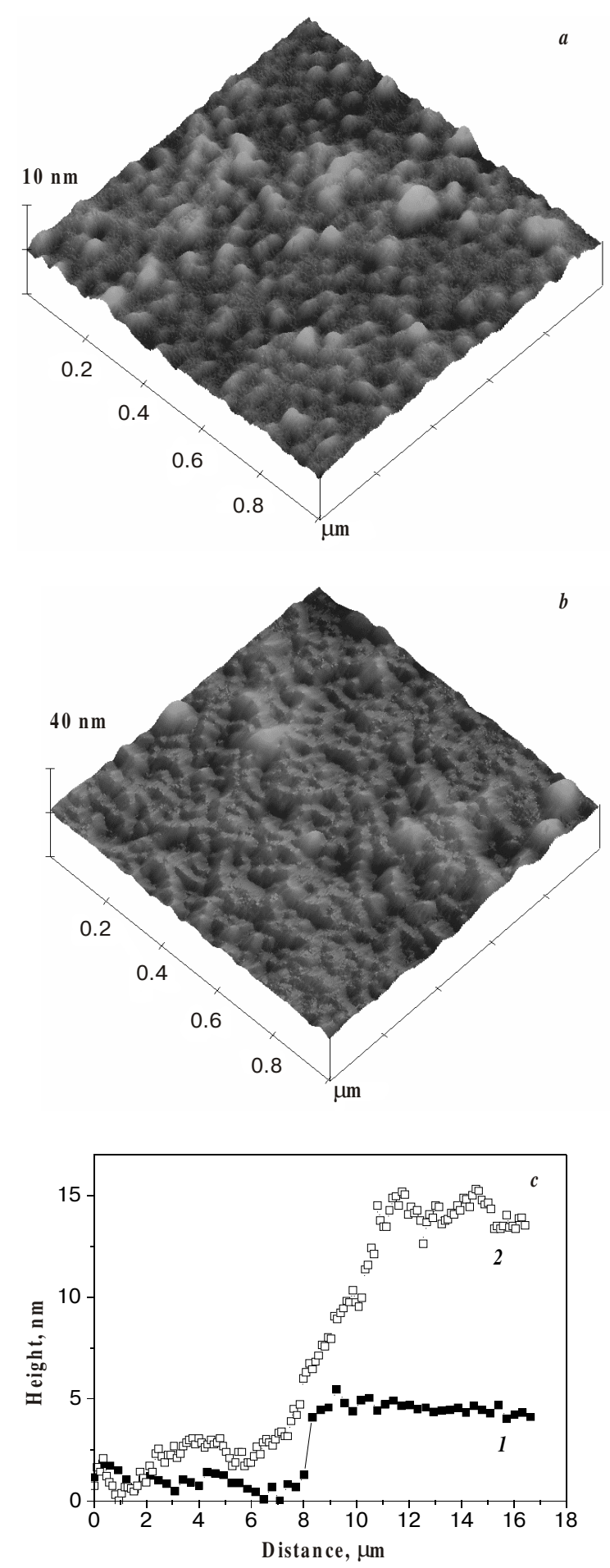

Fig. 1. Three dimensional AFM images $(1 \times 1 \mathrm{~mm})$ for $\mathrm{Au}$ films with mass thickness of $5(a)$ and $(b) 15 \mathrm{~nm}$, and its height profiles in the area between films and substrate $(c)$.

data with approaches 1 or -1 indicating a very strong correlation between two of the parameters in the model were obtained for the standard mode. Therefore, in this case, in order to unambiguously determine the optical constants, the thickness were set equal to the average value 
N.L. Dmitruk et al.: Optical characterization of thin Au films by ...

Table 1. Two-parameter correlation coefficients for Au films, investigated in different ellipsometric modes.

\begin{tabular}{l|c|c|c|c|c}
\hline \hline \multirow{2}{*}{ Samples } & \multirow{2}{*}{$d, \mathrm{~nm}$} & \multirow{2}{*}{ Correlated parameters } & \multicolumn{2}{|c|}{ ATR } & \multicolumn{2}{c}{ Standard } \\
\cline { 3 - 6 } & & $n-d$ & -0.89 & -0.99 & 1.0 \\
\hline 1 & 5.0 & $n-k$ & 0.89 & 0.99 & -1.0 \\
\hline & & $k-d$ & -0.99 & -1.0 & -1.0 \\
\hline 2 & & $n-d$ & -0.90 & -0.98 & 1.0 \\
\hline 3 & 15.0 & $n-k$ & -0.68 & 0.98 & -1.0 \\
\hline & & $k-d$ & 0.74 & -1.0 & -1.0 \\
\hline & 50.0 & $n-d$ & -0.66 & 0.47 & 1.0 \\
\hline \hline & & $n-k$ & -0.10 & -0.47 & -0.99 \\
\hline
\end{tabular}
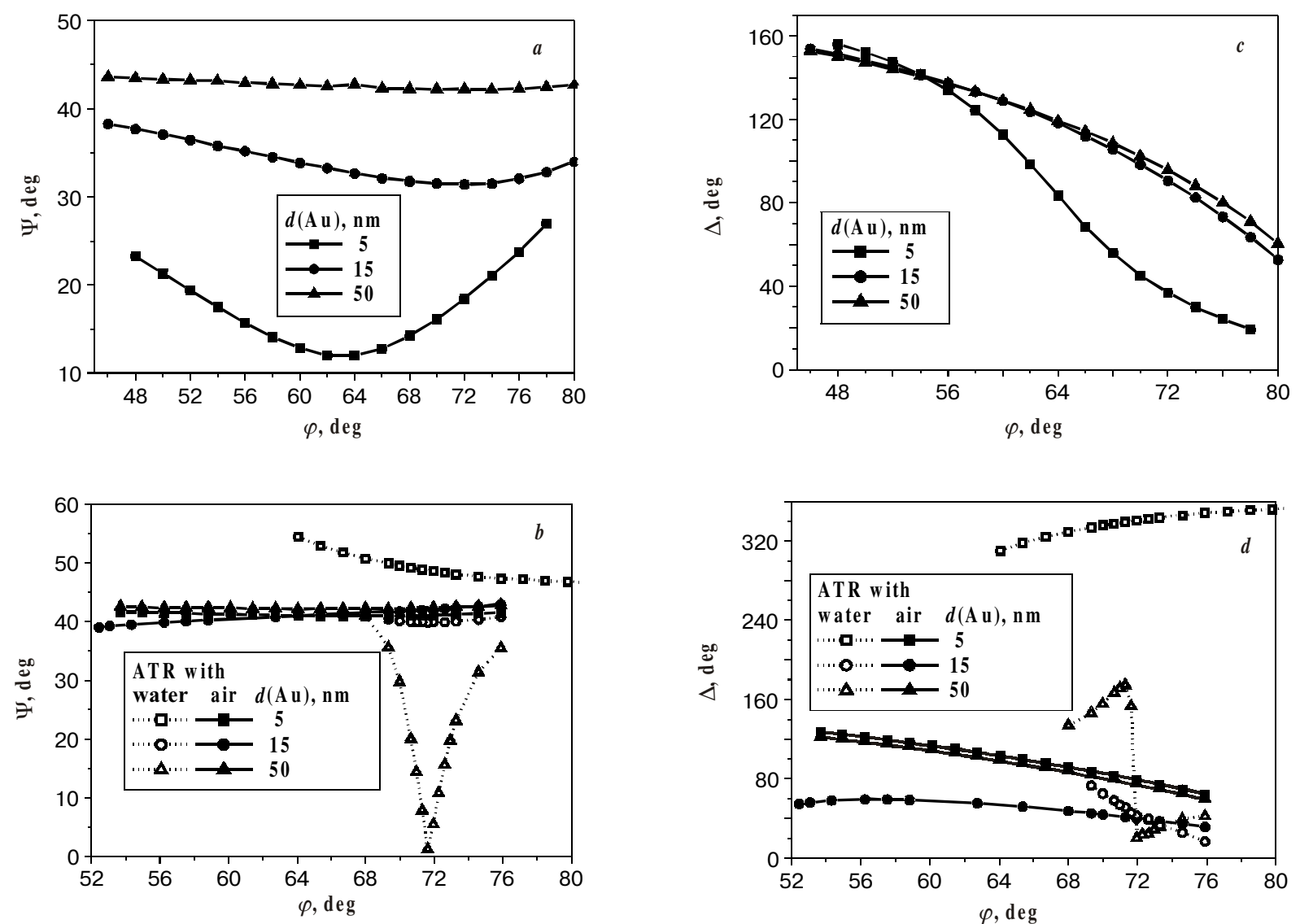

Fig. 2. The $\Psi(\varphi)$ and $\Delta(\varphi)$ experimental dependencies for structures $A u(5,15,50 \mathrm{~nm}) /$ glass, measured in the standard $(a)$ and the ATR $(b)$ ellipsometric modes with water and air media.

of those obtained in ATR measurements. The problem of uniqueness in the case of ultrathin films becomes essential even in the case of ATR mode due to the strong correlation between some parameters (see, Table 2: $k-d, n-d$ ). In this case, the additional experimental techniques for thickness determination increase the reliability of obtained results. In our work, we combined the ellipsometry with AFM in such cases (Fig. 1a).
The values of $n, k$ and $d$ with $90 \%$ confidence limits, where the true parameter value lies with a $90 \%$ probability, in both measurement regimes are shown in Table 2. The obtained results show the thickness dependence of 'effective' optical parameters of Au films. The refractive index decrease, extinction coefficients first increase and then decrease with increasing film thickness. This behavior is typical for metal films [2, 3] with different film density (non-uniformity or granularity) and was qualitatively predicted 
N.L. Dmitruk et al.: Optical characterization of thin Au films by ...

Table 2. Optical parameters and thickness of Au films with $90 \%$ confidence limit in standard and ATR modes of ellipsometry.

\begin{tabular}{l|c|c|l|l|c|c|c|c|c}
\hline \hline \multirow{2}{*}{ Samples } & \multicolumn{4}{c|}{ ATR } & \multicolumn{3}{c}{ Standard } \\
\cline { 2 - 10 } & \multicolumn{2}{|c|}{ Water $(n=1.333)$} & \multicolumn{2}{c|}{ Air $(n=1.0)$} & \multicolumn{3}{c}{ Air $(n=1.0)$} \\
\cline { 2 - 10 } & $n$ & $k$ & $d, \mathrm{~nm}$ & $N$ & $k$ & $d, \mathrm{~nm}$ & $n$ & $k$ & $d, \mathrm{~nm}$ \\
\hline 1 & $1.02 \pm 0.02$ & $2.88 \pm 0.23$ & $3.5 \pm 0.3$ & $1.57 \pm 2.22$ & $2.95 \pm 4.63$ & $4.0 \pm 9.16$ & $1.0 \pm 0.008$ & $3.03 \pm 0.008$ & 4.0 \\
\hline 2 & $0.38 \pm 0.024$ & $3.98 \pm 0.014$ & $13.72 \pm 0.34$ & $0.34 \pm 0.03$ & $3.88 \pm 0.28$ & $13.0 \pm 1.9$ & $0.12 \pm 0.01$ & $3.82 \pm 0.01$ & 14.0 \\
\hline 3 & $0.3 \pm 0.005$ & $3.55 \pm 0.001$ & $49.05 \pm 0.21$ & $0.33 \pm 0.007$ & $3.49 \pm 0.06$ & $49.0 \pm 2.43$ & $0.08 \pm 0.01$ & $3.12 \pm 0.01$ & 50.0 \\
\hline \hline
\end{tabular}

by the effective-medium theory [11]. Second, the optical parameters were higher in the ATR mode than those in standard mode. Third, in ATR mode the optical constants of films with a constant surface structure changed as a function of the refraction index inherent to contact media. To investigate this fact in more detail, the ATR measurements were performed for $45 \mathrm{~nm}$ thick Au films in contact with liquids (water, $20 \%$ and $100 \%$ aqueous solution of alcohol). The experimental dependencies of $\Psi(\varphi)$ and $\Delta(\varphi)$ are shown in Fig. 3. With increasing refractive index of adjacent media the ATR minimum is shifted to higher angle values. It corresponds to decreasing $n$ and
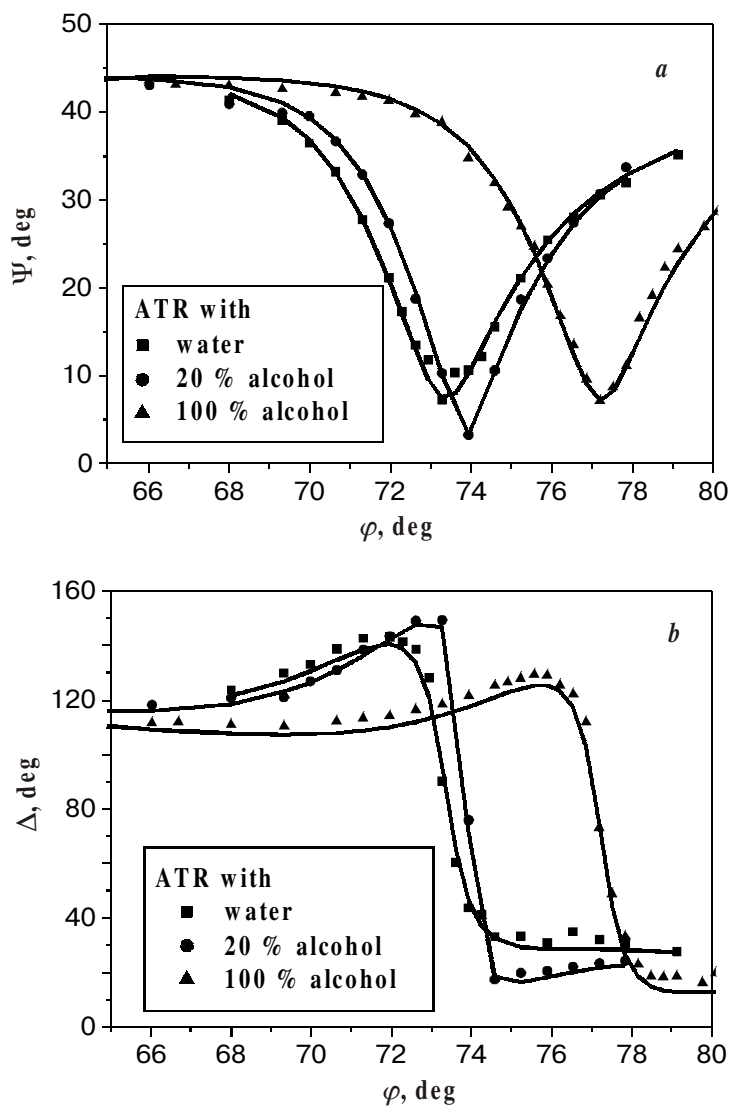

Fig. 3. The $\Psi(\varphi)$ and $\Delta(\varphi)$ experimental (symbols) and calculated (lines) dependencies for the $45 \mathrm{~nm} \mathrm{Au}$ film, measured in the ATR mode immersed with water $(n=1.333), 20 \%$ alcohol $(n=1.345)$ and $100 \%$ alcohol $(n=1.361)$. increasing $k$ values of Au film (Fig. 4). This effect is nonmonotonic with increasing refractive index of contacting media. In general, the difference in the obtained values for the extinction coefficient was in the range of $1-2 \%$, but for the refractive index of about $20 \%$.

The last results agree well with those described by other authors [8,9] in a liquid contacting situation. They found changes of the real part of the dielectric function to less negative values with increasing refractive index of the dielectric media. It follows also from our results. Several mechanisms had been proposed to describe the difference in metal optical constants depending on the refractive index of the ambient medium [8,9]. First one [8] is to consider the surface roughness layer of $\mathrm{Au}$ films as a metal-insulator composite layer according to the Maxwell-Garnett theory [11]. Another one, proposed in [9], is the model of a full porous metal layer by an uniform film with uncompleted volume fraction. In this case, the influence of ambient medium that leads to penetration of the surrounding substance into the voids of the film due to presence of grain boundaries is more probable. The measured changes in the optical constants are consistent with changes of volume fraction.

More experiments need to be carried out to identify the physical process and chemical reaction in every application. The corresponding effects must be taken into consideration in polaritonic optoelectronic and optochemical sensor technique.

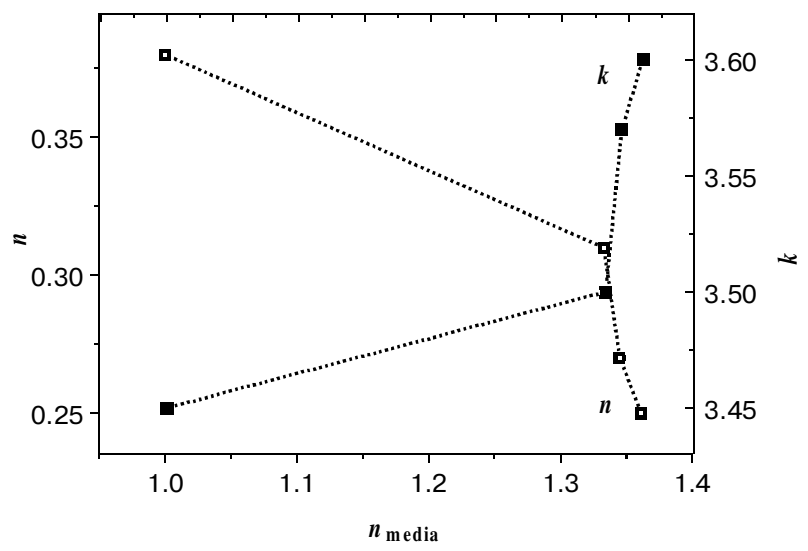

Fig. 4. Optical parameters ( $n$ and $k$ ) of $45 \mathrm{~nm}$ thick Au film calculated from dependencies shown in Fig. 3 vs the refractive index of adjacent media. 


\section{Conclusions}

The determination of effective optical constants and thickness of Au films in the range up to $50 \mathrm{~nm}$ was performed by MAI ellipsometry in the standard and in the ATR modes in contact with various dielectric media. It was shown that unambiguous results were obtained in ATR mode. The mode- and medium- related optical constants of Au films were obtained. It was found that in ATR mode the effective optical parameters were higher than in the standard mode. In ATR mode the refractive index decreases and the extinction coefficient increases with increasing the refractive index of adjoining medium. This effect must be taken into consideration in polaritonic optoelectronics and optochemical sensor technique.

\section{References}

1. D.W. Lynch and W.R. Hunter, In: E.D. Palik (Ed.) // Handbook of Optical Constants of Solids, Academic Press, 1985, pp. 286-295.

2. C. Reale // Infrared Physics, 10, p. 175 (1970).

3. J.J. Xu, J.F. Tang // Applied Optics, 28, p. 2925 (1989).

4. F. Abeles // Surf. Sci., 1, p. 56 (1976).

5. N.L. Dmitruk, V.G. Litovchenko, V.L. Strizhevskyi, Surface Polaritons in Semiconductors and Dielectrics, Naukova Dumka, Kiev, 1989 (in Russian).

6. N.L. Dmitruk, O.Yu. Borkovskaya, O.V. Fursenko, Optoelectronics // Instrumentation and Data Processing, 1, p. 51 (1997).

7. E.G. Bortchagovsky // SPIE Proc., 3094, p. 239 (1997).

8. H. Gugger, M. Jurich, J.D. Swalen // Phys. Rev., B30, p. 4189 (1984).

9. Liang-Yao Chen, D.W. Lynch // Phys. Rev., B36, p. 1425 (1987).

10. G.H. Bu-Abbud, N.M. Bashara, J.A. Woollam // Thin Solid Films, 138 p. 27 (1986).

11. O.S. Heavens, Optical Properties of Thin Solid Films, Dover, New York, 1965, pp. 177-180. 